\title{
THEORETICAL AND EXPERIMENTAL INVESTIGATIONS OF THE BIFURCATION BEHAVIOR OF CREEP GROAN OF AUTOMOTIVE DISK BRAKES
}

\author{
Xingwei Zhao, Nils Gräbner, Utz von Wagner \\ Technische Universität Berlin, Chair of Mechatronics and Machine Dynamics (MMD), Berlin, Germany \\ e-mail: utz.vonwagner@tu-berlin.de
}

\begin{abstract}
There are several low frequency vibration phenomena which can be observed in automotive disk brakes. Creep groan is one of them provoking noise and structural vibrations of the car. In contrast to other vibration phenomena like brake squeal, creep groan is caused by the stick-slip-effect. A fundamental investigation of creep groan is proposed in this paper theoretically and experimentally with respect to parameter regions of the occurrence. Creep groan limit cycles are observed while performing experiments in a test rig with an idealized brake. A nonlinear model using the bristle friction law is set up in order to simulate the limit cycle of creep groan. As a result, the system shows three regions of qualitatively different behavior depending on the brake pressure and driving speed, i.e. a region with a stable equilibrium solution and a stable limit cycle, a region with only a stable equilibrium solution, and a region with only a stable limit cycle. The limit cycle can be interpreted as creep groan while the equilibrium solution is the desired vibration-free case. These three regions and the bifurcation behavior are demonstrated by the corresponding map. The experimental results are analyzed and compared with the simulation results showing good agreement. The bifurcation behavior and the corresponding map with three different regions are also confirmed by the experimental results. At the end, a similar map with the three regions is also measured at a test rig with a complete real brake.
\end{abstract}

Keywords: creep groan, stick-slip limit cycle, equilibrium solution, bifurcation

\section{Introduction}

Creep groan is a low frequency vibration phenomenon in automotive disk brakes occurring in particular, when the brake is slowly released while the car starts moving (Abdelhamid, 1995; Abdelhamid and Bray, 2009; Zhao et al., 2016, 2017; Brecht et al., 1997; Brecht, 2000; Crowther and Singh, 2007, 2008). The frequency range is up to $500 \mathrm{~Hz}$ and it is caused by the stick-slipeffect. Like other brake noise problems, such as brake squeal (Cantoni et al., 2009; von Wagner et al., 2007), creep groan is mainly a comfort problem resulting in possible warranty claims and additional costs for the manufacturer.

The simplest usual way to describe the stick-slip-effect is to use Coulomb's friction law. A general study on friction induced vibrations has been performed by Ibrahim giving also an overview about contact mechanics and friction forces (Ibrahim, 1994a,b). With respect to creep groan, Brecht et al. (1997) and Brecht (2000) measured its vibration characteristics and studied the corresponding stick-slip limit cycle. Crowther and Singh (2007, 2008) modeled the creep groan phenomenon in terms of two dynamic sub-systems coupled with Coulomb's friction law. Vadari and Jackson (1999) considered the relationship of creep groan to vehicle dynamics by looking at the suspension response. A more complex nonlinear model with 7 degree-of-freedom (DOF) with Coulomb's friction law was utilized for studying the mechanism of creep groan by Wu and Jin (2014). 
However, the models using Coulomb's friction law cannot explain all effects occurring during creep groan. In order to overcome this deficiency, Hetzler et al. (2007) presented an analytical investigation on stability and bifurcation behavior of stick-slip motions, where a friction coefficient depended on the relative velocity between the two contact partners.

Since friction induced vibrations are naturally highly related to the frictional contact, some works are concentrating on studying the characteristics of the involved pad materials. A study performed by Jang et al. (2001) on the creep groan propensity of different friction materials resulted in the conclusion that creep groan can be reduced when the friction material contains a small difference between the static and dynamic friction coefficients. Similar results can be obtained when considering elementary friction oscillators. Fuadi et al. (2009, 2010) studied creep groan by considering a caliper-slider experimental model, and derived a map showing necessary conditions for avoiding creep groan. Results of creep groan vehicle tests were shown by Neis et al. (2016) and their relation with stick-slip in laboratory tests was investigated. However, most of those works were experimental investigations, and a model showing high agreement with experimental results is still to be developed.

The present paper extends the prior work of the same authors (Zhao et al., 2016, 2017) performing the fundamental work with minimal models for creep groan using the bristle friction law. Creep groan is first measured in a test rig with an idealized brake, which is in most parts similar to a real brake system but with less hard-to-model components. After that, creep groan is studied fundamentally through (compared to Zhao et al., 2016, 2017) an extended minimal model using also the bristle friction law. The bristle friction law proposed by De Wit et al. (1995) and Johanastrom and De Wit (2008) includes the Stribeck effect, hysteresis and springlike characteristics for stick. By analyzing the bifurcation behavior of the resulting nonlinear model, three regions of qualitatively different behavior depending on the parameters can be identified. The main focus of this paper is to describe the detailed bifurcation behavior and the corresponding map with three different regions according to the occurrence and absence of creep groan, while experiments testified the existence of them in the test rig with the idealized brake. Furthermore, a similar map is also measured from the test rig with a real brake.

\section{Test rig with an idealized brake}

For the experimental investigation of creep groan, a test rig with an idealized brake has been designed and assembled at MMD TU Berlin. This set-up (Fig. 1) imitates the brake system of an automotive vehicle. The intention of the design was to concentrate on the pad-disk friction contact and to avoid other hard-to-model parts. In the real brake, there are additional hardto-model parts, such as complicated structure of the carrier and its coupling with the caliper via bushings. These parts may also have an influence on the creep groan behavior but are considered to have minor influence compared to the frictional contact between the pad and the disk. Therefore, a carrier consisting of two L-shaped steel plates with high stiffness in the in-plane direction (but comparable order of stiffness similar to the real brake carrier) and low stiffness in the out-of-plane direction replaces the real brake carrier. The pads (from a serial brake) are fixed on the long legs of the carrier while its short legs are fixed to the base plate. A caliper from the same serial brake is attached to the long legs of the carrier providing the pressure force. The disk is driven by an AC motor coupled with a reduction gearbox, which can provide a low revolution speed (starting from $0.1 \mathrm{rad} / \mathrm{s}$ i.e. approximately $0.12 \mathrm{~km} / \mathrm{h}$ ) with a high torque. A drive shaft connects the gear box and the brake disk. This set-up is almost similar with a real brake system, since the pads, the disk, the drive shaft and the caliper of the test rig are taken from a real vehicle. Moreover, the set-up has advantages for the experimental investigation of creep groan, such as: 1 . The set-up has a simple structure of the components and, therefore, its 
parameters are easy to identify; 2 . The set-up is easy to assemble with different types of sensors since the disk is directly driven by the shaft. In contrast to this, in industrial dynos, the disk is usually driven via wheel bolts, which complicates accessibility to the brake for measurements.

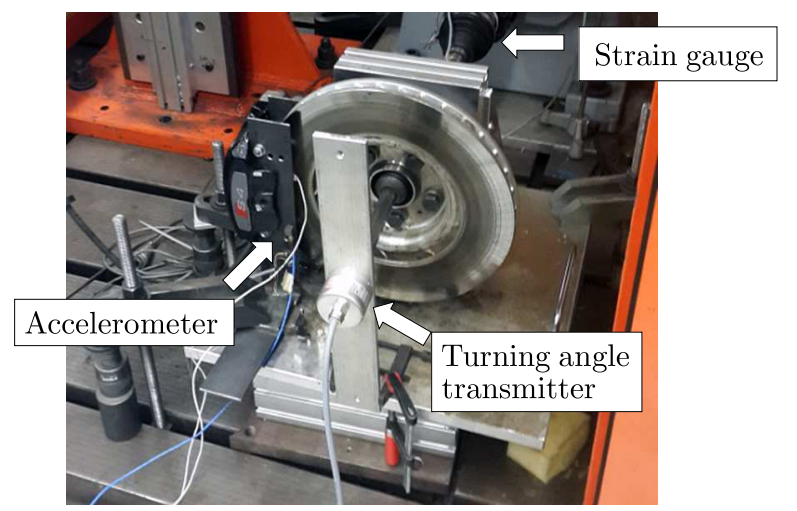

Fig. 1. Test rig with an idealized brake at MMD TU Berlin

The following sensors have been assembled to the set-up. A strain sensor is placed on the drive shaft measuring its torsional angle. It is calibrated through a static measurement (Muschalle, 2015). A turning angle transmitter (power supply $5 \mathrm{~V} \mathrm{DC}$, maximum sample rate $40 \mathrm{kHz}$, resolution $14 \mathrm{bit}$ ) is connected to the disk which allows for the measurement of the absolute angle and angular velocity of the disk. The pressure of the brake can be read from a pressure meter. An accelerometer (PCB $4507 \mathrm{ICP}$ accelerometers, frequency range $0.3 \mathrm{~Hz}-6 \mathrm{kHz}$, sensitivity $101.2 \mathrm{mV} / \mathrm{g}$ ) is attached to the long leg of the carrier, which measures the acceleration of the pad in the vertical direction. The measured signals are conditioned and subsequently driven into a data acquisition module (featuring eight 24-bit simultaneously sampled A/D channels, maximum sample rate $80 \mathrm{kHz}$, and analogue anti-aliasing filter).

The measured torsional angle $\Delta \theta$ of the shaft describing the difference between the rotation angle of the drive and the rotation angle of the disk (defined in Section 3), and the pad acceleration $\ddot{x}$ are displayed in Fig. 2 without creep groan and Fig. 3 in the case of creep groan. One can differ the creep groan case from the non-creep groan case, where $\Delta \theta$ has large vibrations and impulses can be found in the acceleration signal $\ddot{x}$ during creep groan. The kurtosis value, which is based on a scaled version of the fourth moment of the signal, can be used to detect creep groan from the non-creep groan case. A higher kurtosis is the result of extreme deviations, which is characteristic for non-stationary signals (Antoni, 2006; Antoni and Randall, 2006). For the non-creep groan case, the kurtosis value of the pad acceleration signal is shown in Fig. 2b is 2.85, while this kurtosis value increases to 7.06 when creep groan occurs as shown in Fig. 3b.
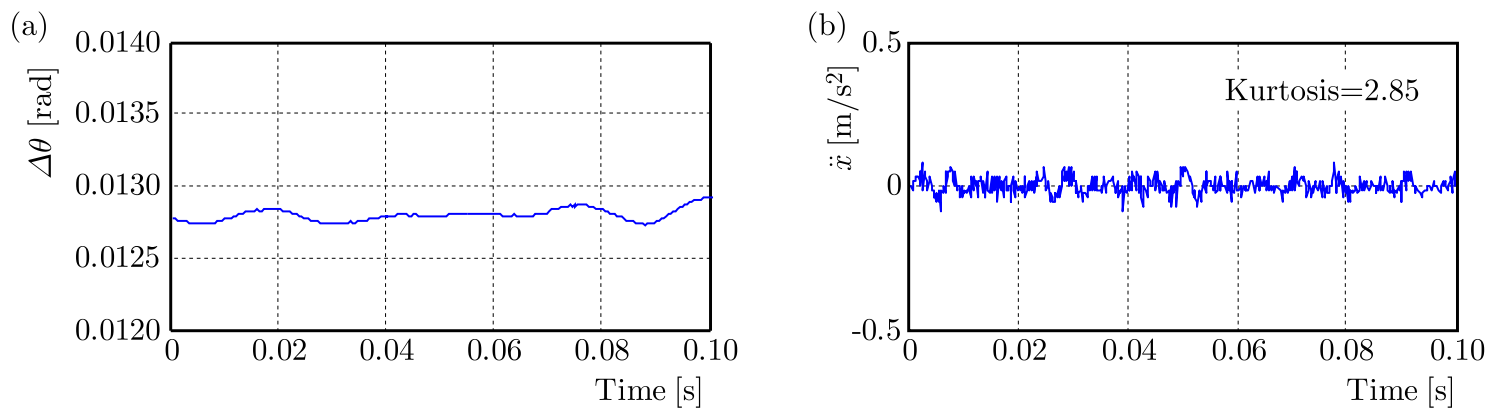

Fig. 2. Torsional angle of the shaft $\Delta \theta$ (a) and acceleration of the pad $\ddot{x}$ (b) without creep groan

The frequency spectra of the signals during creep groan are exhibited in Fig. 3c,d by pursued Discrete Fourier transformations. The set-up consists of non-rotating parts in terms of the brake 
(a)

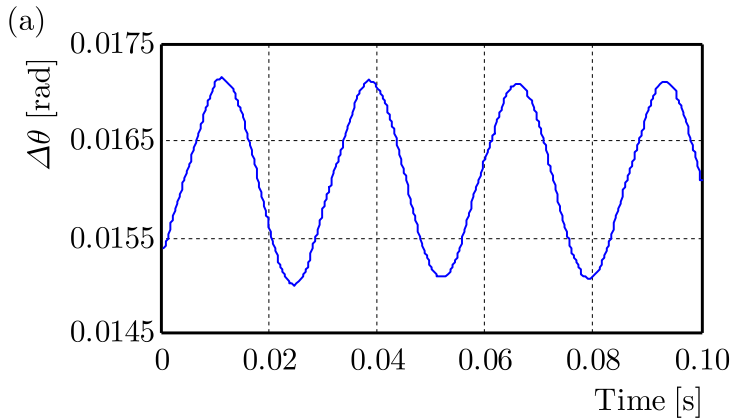

(c)

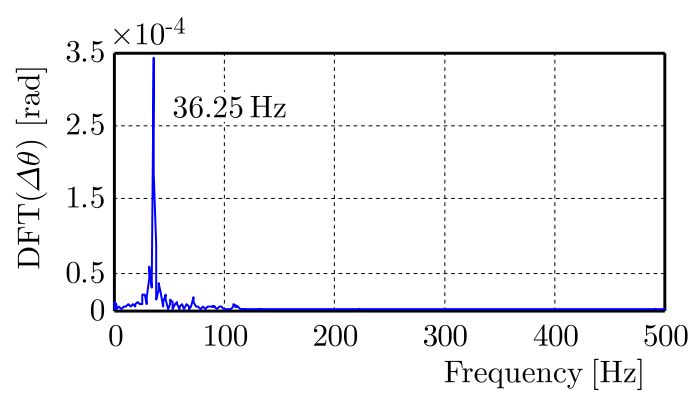

(b)

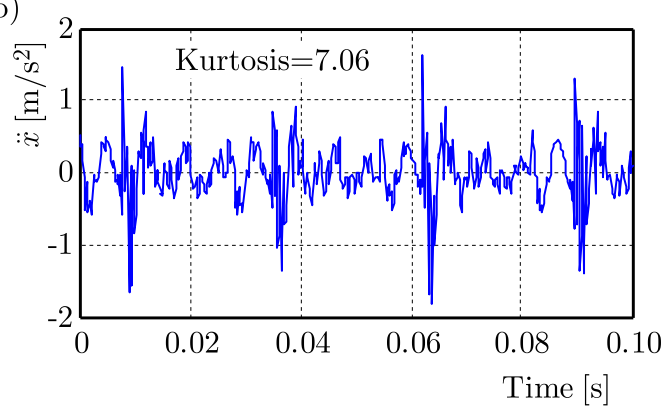

(d)

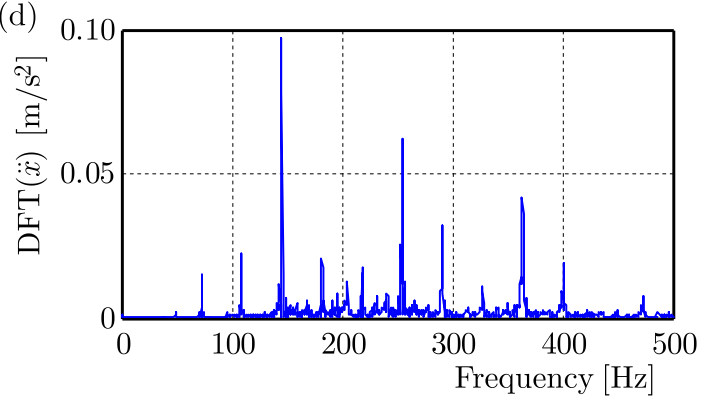

Fig. 3. Torsional angle of the shaft $\Delta \theta$ (a) and acceleration of the pads $\ddot{x}$ (b), and their frequency spectra (c), (d) during creep groan

pads, the caliper, and the carrier, as well as rotating parts in terms of the disk, the drive shaft and the motor. In the rotating parts, the spectrum of $\Delta \theta$ shows a single peak at $36.25 \mathrm{~Hz}$, which is the frequency related to the period of the stick-slip motion. In the non-rotating parts, the spectrum of $\ddot{x}$ shows a lot of frequency peaks, also at much higher frequencies than the frequency of the stick-slip motion, and this vibration is what the human hear/feel during creep groan. According to the measured signals from both parts, it is clear that stick-slip happens during creep groan, and the creep groan is the resulted vibration of the brake system.

\section{Fundamental theoretical investigations of creep groan}

The chosen minimal model for the investigation of creep groan is shown in Fig. 4. It is considered as a two DOF system. Minimal models for creep groan with comparable low number of degrees of freedom are, as already mentioned in the introduction, discussed by Brecht (2000) with three degrees of freedom, in (von Wagner et al., 2007) and (Ibrahim, 1994a) by Crowther and Singh (2007, 2009) with four degrees of freedom or by Fuadi et al. (2010) with two degrees of freedom. With our model, we focus on the stability and bifurcation analysis of the nonlinear model in comparison with our experimental results.

As we are in the low frequency range, the disk is considered as a rigid body which is connected to the motor by the drive shaft. During creep groan, the pads and the caliper move synchronously. Thus, the brake pads together with the caliper are considered as a rigid body and are connected to the base frame through the carrier. As the disk can only perform rotation but no wobbling motion, the number of pads can be reduced from two to one without any influence on the qualitative behavior of the model. The drive shaft is modeled as a rotational spring with stiffness $k_{\theta}$ and damping $d_{\theta}$. With $\theta$ being the disk rotation angle and $\Omega_{0} t$ the rotation angle of the drive with constant $\Omega_{0}$, the equation of motion of the disk is given by

$$
I \ddot{\theta}+d_{\theta}\left(\dot{\theta}-\Omega_{0}\right)+k_{\theta}\left(\theta-\Omega_{0} t\right)=-F_{R} r
$$

where $r$ is the radius of the point the pads act on the disk, $I$ is the moment of inertia of the disk and $F_{R}$ is the friction force in the contact between the disk and pads. Introducing $\Delta \theta$ as 
the difference between the rotation angle of the drive $\Omega_{0} t$ and the disk rotation angle $\theta$, with $\Delta \theta=\Omega_{0} t-\theta$, this equation of motion of the disk reads

$$
I \Delta \ddot{\theta}+d_{\theta} \Delta \dot{\theta}+k_{\theta} \Delta \theta=F_{R} r
$$

The carrier is considered as a spring with stiffness $k_{x}$ and damping $d_{x}, m$ is the mass of the pads with the caliper, and $x$ is the displacement of the pads. The equations of motion of the system can then be described by

$$
\begin{array}{lc}
\mathbf{M} \ddot{\mathbf{Y}}+\mathbf{C} \dot{\mathbf{Y}}+\mathbf{K Y}=\mathbf{F} & \mathbf{M}=\left[\begin{array}{cc}
I & 0 \\
0 & m
\end{array}\right] \\
\mathbf{Y}=\left[\begin{array}{c}
\Delta \theta \\
x
\end{array}\right] & \mathbf{C}=\left[\begin{array}{cc}
d_{\theta} & 0 \\
0 & d_{x}
\end{array}\right] \\
\mathbf{K}=\left[\begin{array}{cc}
k_{\theta} & 0 \\
0 & k_{x}
\end{array}\right] & \mathbf{F}=\left[\begin{array}{c}
F_{R} r \\
F_{R}
\end{array}\right]
\end{array}
$$

Due to its definition, $\Delta \theta$ has in general due to the influence of the friction force a positive nonzero mean value.
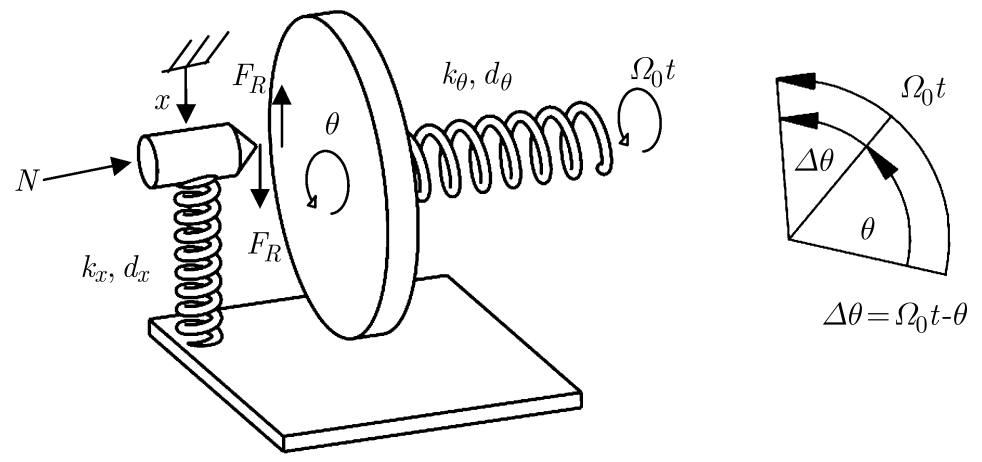

Fig. 4. Model of the test rig with an idealized brake

For the determination of $F_{R}$, the bristle friction law is used in the following. By imagining that two rigid bodies are in contact through elastic bristle surfaces (De Wit et al., 1995; Johanastrom and De Wit, 2008), the friction force $F_{R}$ is generated by deformation of the bristle. The dynamic friction force $F_{R}$ can in general be expressed as

$$
F_{R}=\sigma_{0} z+\sigma_{1} \dot{z}
$$

where $\sigma_{0}$ is the contact stiffness coefficient, $\sigma_{1}$ is the contact damping coefficient. $z$ is the average deflection of the bristles described by

$$
\dot{z}=\phi(\Delta \dot{\theta}, \dot{x}, z)=\Omega_{0} r-\Delta \dot{\theta} r-\dot{x}-\frac{z}{g_{0}}\left|\Omega_{0} r-\Delta \dot{\theta} r-\dot{x}\right|
$$

Herein, $g_{0}$ is a scaling factor which includes the Stribeck effect. $g_{0}$ is given as

$$
g_{0}=\frac{1}{\sigma_{0}}\left(N \mu_{d}+N\left(\mu_{s}-\mu_{d}\right) \exp \left(-\left|\frac{\left(\Omega_{0}-\Delta \dot{\theta}\right) r-\dot{x}}{v_{s}}\right|^{\alpha}\right)\right)
$$

where $v_{s}$ is Stribeck velocity, $N$ is the brake normal force, $\mu_{s}$ and $\mu_{d}$ are the static and dynamic friction coefficients, $\alpha$ is an empirical parameter which can be measured in experiments. In our test rig, the value $\alpha=1$ is chosen as the more reasonable approach (Johanastrom and De Wit, 2008; Tustin, 1947), since $\alpha=1$ leads to better agreement between experimental and simulation 
results. The bristle friction law can be described in such a way that the bristles will deflect like springs at a tangential force, which gives rise to the friction force. If the force is so large that some of the bristles deflect, slip occurs. This bristle friction law includes the Stribeck effect, hysteresis, pre-sliding characteristics of friction. The complete dynamic equations of the entire model can be, therefore, written as a set of first order ordinary differential equations

$$
\begin{aligned}
& \dot{\mathbf{Y}}=\left[\begin{array}{c}
\Delta \dot{\theta} \\
\dot{x} \\
-\frac{d_{\theta}}{I} \Delta \dot{\theta}-\frac{k_{\theta}}{I} \Delta \theta+\frac{r}{I} \sigma_{0} z+\frac{r}{I} \sigma_{1} \phi(\Delta \dot{\theta}, \dot{y}, z) \\
-\frac{d_{x}}{m} \dot{x}-\frac{k_{x}}{m} x+\frac{1}{m} \sigma_{0} z+\frac{1}{m} \sigma_{1} \phi(\Delta \dot{\theta}, \dot{y}, z) \\
\phi(\Delta \dot{\theta}, \dot{y}, z)
\end{array}\right] \\
& \mathbf{Y}=\left[\begin{array}{lllll}
\Delta \theta & x & \Delta \dot{\theta} & \dot{x} & z
\end{array}\right]^{\mathrm{T}} \\
& \phi(\Delta \dot{\theta}, \dot{y}, z)=\Omega_{0} r-\Delta \dot{\theta} r-\dot{x}-\frac{z}{g_{0}}\left|\Omega_{0} r-\Delta \dot{\theta} r-\dot{x}\right| \\
& g_{0}=\frac{1}{\sigma_{0}}\left(N \mu_{d}+N\left(\mu_{s}-\mu_{d}\right) \exp \left(-\left|\frac{\left(\Omega_{0}-\Delta \dot{\theta}\right) r-\dot{x}}{v_{s}}\right|^{\alpha}\right)\right)
\end{aligned}
$$

where $\mathbf{Y}$ is the vector of state variables of the system.

The equilibrium solution of equations (3.7), which is the equilibrium position of the system, is expressed as

$$
\begin{aligned}
\dot{\mathbf{Y}}_{e q} & =\mathbf{0} \\
\mathbf{Y}_{e q} & =\left[\begin{array}{lllll}
\frac{r \mathcal{A}}{k_{\theta}} & 0 & \frac{\mathcal{A}}{k_{x}} & 0 & \frac{\mathcal{A}}{\sigma_{0}}
\end{array}\right]^{\mathrm{T}}
\end{aligned}
$$

where $\mathcal{A}=N \mu_{d}+N\left(\mu_{s}-\mu_{d}\right) \mathrm{e}^{-\Omega_{0} r / v_{s}}$.

Linearizing equation $(3.7)_{1}$ under its equilibrium position, we get

$$
\begin{aligned}
\dot{Y} & =\mathbf{A Y} \\
\mathbf{A} & =\left[\begin{array}{ccccc}
0 & 1 & 0 & 0 & 0 \\
-\frac{k_{\theta}}{I} & -\frac{d_{\theta}}{I}+\frac{r \sigma_{1}}{I} \frac{\partial \widetilde{\phi}}{\partial \Delta \dot{\theta}} & 0 & \frac{r \sigma_{1}}{I} \frac{\partial \widetilde{\phi}}{\partial \dot{x}} & \frac{r \sigma_{0}}{I}+\frac{r \sigma_{1}}{I} \frac{\partial \widetilde{\phi}}{\partial z} \\
0 & 0 & 0 & 1 & 0 \\
0 & \frac{\sigma_{1}}{m} \frac{\partial \widetilde{\phi}}{\partial \Delta \dot{\theta}} & -\frac{k_{x}}{m} & -\frac{d_{x}}{m}+\frac{\sigma_{1}}{m} \frac{\partial \widetilde{\phi}}{\partial \dot{x}} & \frac{\sigma_{0}}{m}+\frac{\sigma_{1}}{m} \frac{\partial \widetilde{\phi}}{\partial z} \\
0 & \frac{\partial \tilde{\phi}}{\partial \Delta \dot{\theta}} & 0 & \frac{\partial \widetilde{\phi}}{\partial \dot{x}} & \frac{\partial \widetilde{\phi}}{\partial z}
\end{array}\right]
\end{aligned}
$$

where

$$
\begin{aligned}
& \frac{\partial \widetilde{\phi}}{\partial \Delta \dot{\theta}}=\frac{\partial \phi(\Delta \dot{\theta}, \dot{x}, z)}{\partial \Delta \dot{\theta}}=\frac{\left(\mu_{s}-\mu_{d}\right) r^{2} \Omega_{0}}{v_{s}\left(\mu_{s}-\mu_{d}+\mu_{d} \mathrm{e}^{\Omega_{0} r / v_{s}}\right)} \\
& \frac{\partial \widetilde{\phi}}{\partial \dot{x}}=\frac{\partial \phi(\Delta \dot{\theta}, \dot{x}, z)}{\partial \dot{x}}=\frac{\left(\mu_{s}-\mu_{d}\right) r \Omega_{0}}{v_{s}\left(\mu_{s}-\mu_{d}+\mu_{d} \mathrm{e}^{\Omega_{0} r / v_{s}}\right)} \\
& \frac{\partial \widetilde{\phi}}{\partial \Delta z}=\frac{\partial \phi(\Delta \dot{\theta}, \dot{x}, z)}{\partial \Delta z}=\frac{-r \sigma_{0} \Omega_{0}}{N \mu_{d}+N\left(\mu_{s}-\mu_{d}\right) \mathrm{e}^{-\Omega_{0} r / v_{s}}}
\end{aligned}
$$


and $\mathbf{A}$ is the corresponding system matrix. The eigenvalue of the matrix can be calculated as

$$
\lambda=\operatorname{eig}(\mathbf{A})
$$

The Lyapunov stability of the equilibrium solution can be analyzed by eigenvalues $\lambda$. The equilibrium solution is asymptotically stable, when the real parts of all the eigenvalues are negative. In contrast, the equilibrium solution is unstable when any of the real parts of eigenvalues is positive. With respect to the nonlinear behavior, we call an isolated periodic solution in a selfexcited system a limit cycle (Hagedorn, 1978). The limit cycle of Eq. (3.7) is calculated in a transient analysis by using numerical integration.

According to the conditions for the existence of the stick-slip limit cycle and the stability of the equilibrium solution, the system has three different regions with different types of solutions. Corresponding results are shown exemplarily in Fig. 5 if both the speed $\Omega_{0}$ and brake pressure $p$ are varied. Detailed comparison with the experimental results is performed in Section 4. When the system has a stable equilibrium solution but no a stick-slip limit cycle, creep groan cannot occur in this parameter region, and this region is labeled as region I (white region in Fig. 5). When a stable stick-slip limit cycle and a stable equilibrium solution exist simultaneously, occurrence or absence of creep groan depends on its initial condition. We labeled this region as region II (green region in Fig. 5). When the system has a stable stick-slip limit cycle and an unstable equilibrium solution, creep groan will always occur, and this region is labeled as region III (yellow region in Fig. 5). Figure 5 exhibits also, for a constant $\Omega_{0}$ with varying $p$ and for a constant $p$ with varying $\Omega_{0}$, respectively, the largest real part of the eigenvalues $\operatorname{Re}(\lambda)$ of the system matrix $\mathbf{A}$ (red line), and the torsional angle limit cycle amplitude $A_{L C}$ (blue line). The boundary between regions II and III is defined as a curve, where the largest real part of the eigenvalues $\operatorname{Re}(\lambda)$ of the system matrix is equal to 0 . The way to determine the boundary between regions I and II is given as follows: for varying brake pressure and speed, the solution of the nonlinear system can be calculated by a numerical time integration of Eq. (3.7) with initial conditions in the stick region. If the solution is still the stick-slip limit cycle after a while, the stick-slip limit cycle is considered to exist and be asymptotically stable. In addition, due to the system with the negative real parts of the eigenvalues, its equilibrium solution is asymptotically stable and we have coexistence of the stable equilibrium solution and the limit cycle, i.e. we are in region II. If the solution diverges from the limit cycle and converges to the equilibrium solution, a stable stick-slip limit cycle is considered not to exist, i.e. we are in region I. By varying the brake pressure and driving speed, regions I and II as well as the boundary between them can be determined.

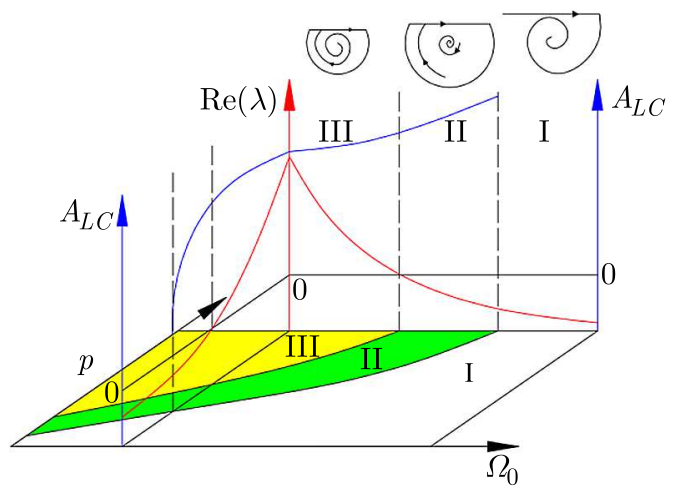

Fig. 5. Map of solution regions depending on the parameters $\Omega_{0}$ and $p$ : I only stable equilibrium solution, II coexistence of the stable equilibrium solution and the limit cycle solution, III only stable limit cycle solution. The largest real part of the eigenvalues $\operatorname{Re}(\lambda)$ of the system matrix $\mathbf{A}$ (red line) and the limit cycle amplitude $A_{L C}$ (blue line) for a constant $\Omega_{0}$ with varying $p$ and for a constant $p$ with varying $\Omega_{0}$, respectively 


\section{Comparison between experimental and simulation results}

Experimental and theoretical results will be compared in this Section. Due to the simple structure of the idealized brake in the test rig, its physical parameters can comparably easy be identified by experiments. The stiffness and damping of the drive shaft and the carrier are determined by modal analyses. The mass of the disk and the caliper with the pads can be measured by a weighting device. The friction coefficients are estimated from comparison of the experimental and simulation limit cycle results. The chosen parameters are given in Table 1.

Table 1. Parameters of the system

\begin{tabular}{|c|c|}
\hline Parameters & Values \\
\hline \hline $\mathrm{I}$ & $0.2025 \mathrm{kgm}^{2}$ \\
\hline$k_{\theta}$ & $1.036 \cdot 10^{4} \mathrm{Nm}$ \\
\hline$d_{\theta}$ & $2 \mathrm{Nms}$ \\
\hline$k_{x}$ & $9.87 \cdot 10^{6} \mathrm{~N} / \mathrm{m}$ \\
\hline$d_{x}$ & $1.5 \cdot 10^{3} \mathrm{Ns} / \mathrm{m}$ \\
\hline$r$ & $0.15 \mathrm{~m}$ \\
\hline$\mu_{d}$ & 0.325 \\
\hline$\mu_{s}-\mu_{d}$ & 0.009 \\
\hline$\sigma_{0}$ & $3.97 \cdot 10^{8} \mathrm{~N} / \mathrm{m}$ \\
\hline$\sigma_{1}$ & $3.84 \cdot 10^{5} \mathrm{Ns} / \mathrm{m}$ \\
\hline$v_{s}$ & $0.025 \mathrm{~m} / \mathrm{s}$ \\
\hline
\end{tabular}

(a)

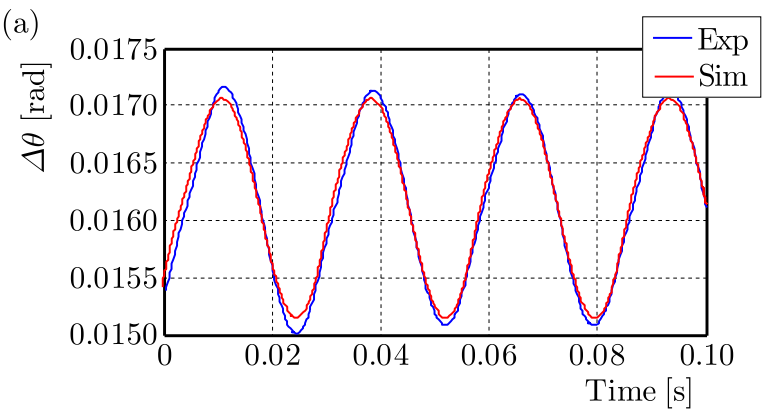

(c)

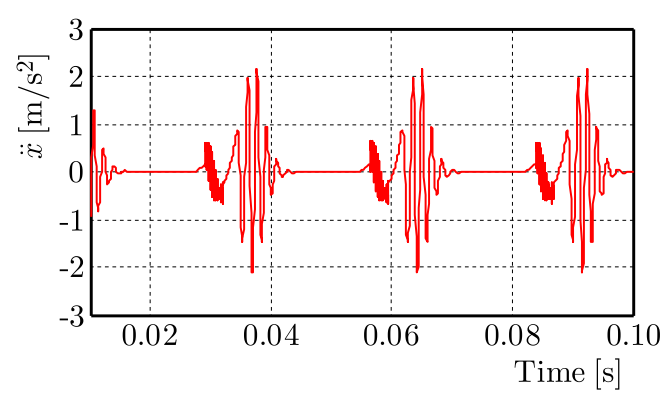

(b)

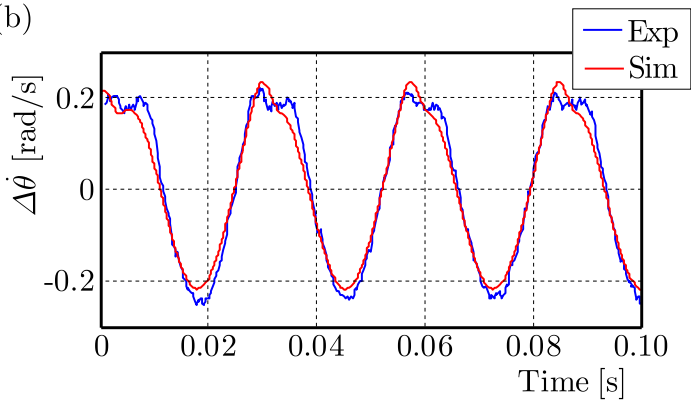

(d)

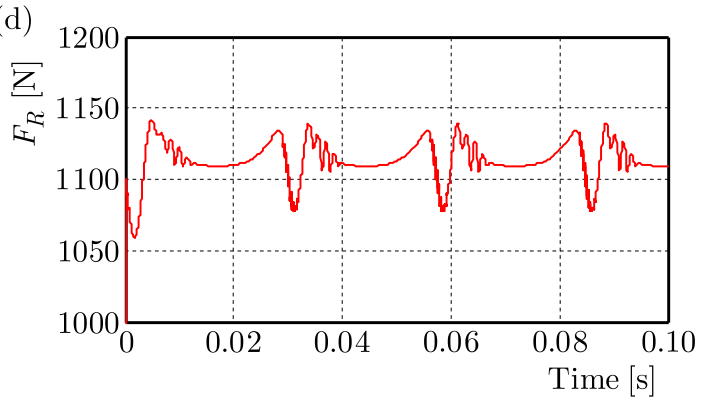

Fig. 6. Simulated and measured torsional angle and torsional velocity of the shaft (a), (b), simulated acceleration of the pad (c) and a simulated friction force (d) with a brake pressure 9 bar and speed $0.2 \mathrm{rad} / \mathrm{s}$

Figures $6 \mathrm{a}$ and $6 \mathrm{~b}$ show the measured and simulated torsional angle and torsional velocity of the drive shaft, with brake pressure 9 bar and driving speed $0.2 \mathrm{rad} / \mathrm{s}$. The red line denotes the simulation results and the blue line presents the experimental results, where both of them show good agreement. The simulated acceleration of the brake pads and the corresponding friction force are proposed in Figs. 6c and 6d. When the system converts from the stick region to the 
slip region, the friction force changes suddenly and a large impulse appears in the acceleration signal. The same effect can also be observed in the measured acceleration on the carrier shown in Fig. 2b. Figure 7 exhibits the simulated and measured stick-slip limit cycles in the phase plot with $\Delta \dot{\theta}$ as a function of $\Delta \theta$. At a constant driving speed, the amplitude of the limit cycle increases with an increase in the brake pressure. The measured and simulated stick-slip limit cycles at 5, 7, and 9 bar are plotted in Fig. 7 .
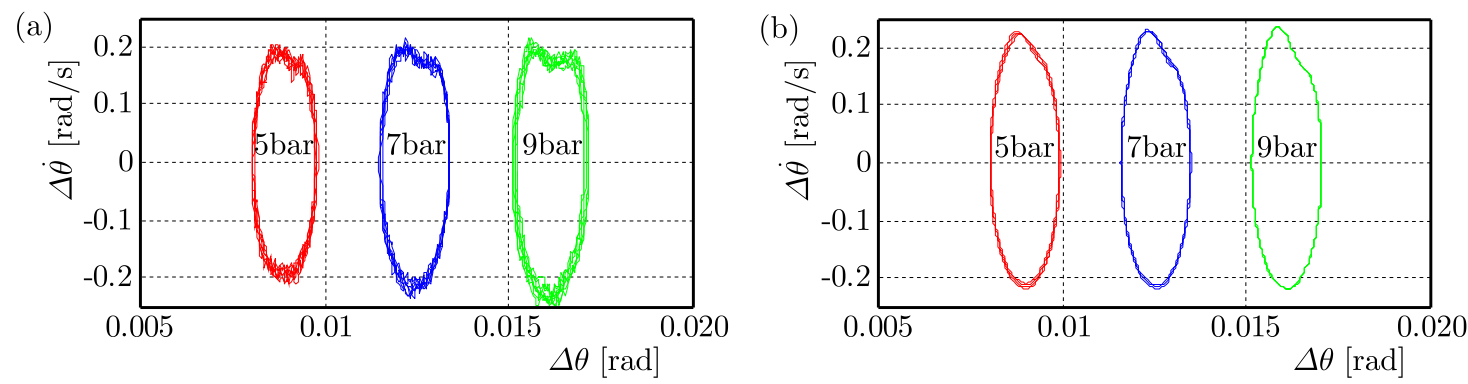

Fig. 7. Limit cycle of experimental results (a) and simulation results (b)

The map of the parameter regions is drawn in Fig. 8 with the identified parameters. Its horizontal axis is the speed of the motor and the vertical axis is the brake pressure, which is proportional to the normal brake force. This map shows different critical conditions of creep groan during accelerating and decelerating of a vehicle. If the operation starts with parameters in region III, creep groan must occur, as the equilibrium solution is unstable. Creep groan will proceed also in region II if we move slowly within this parameter map until the system enters in parameter region I, since the limit cycle does not exist in region I. Following the dotted line in Fig. 8, this is usually the case when the vehicle is accelerated. If the vehicle is decelerated, different things will happen, as shown in Fig. 8 with the solid line. In region I, the silent solution without creep groan is the only stable solution. The system will stay in the attractor of the silent solution even though it enters region II, and no creep groan is occurring. Creep groan will then occur once region III is entered, as the limit cycle is the only stable solution. This can explain that creep groan is more serious in the accelerating process than in the decelerating process, which agrees with the driving experience.

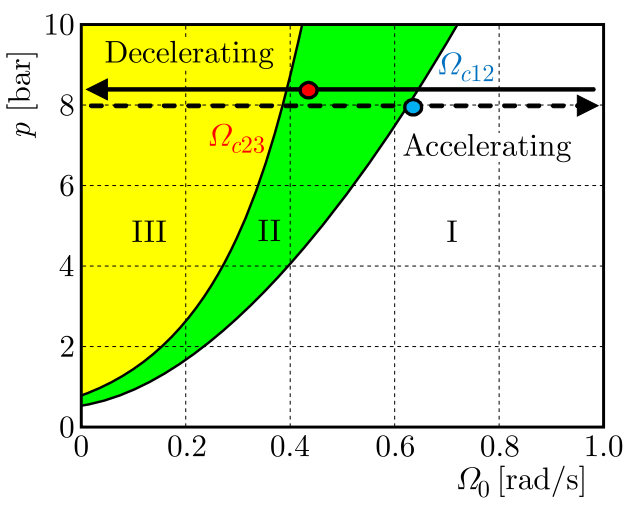

Fig. 8. Map of creep groan, the red point represents the measured boundary between regions II (green) and III (yellow), while the blue point represents the measured boundary between regions I (white) and II (green)

The determination of the critical velocities $\Omega_{c 12}$ and $\Omega_{c 23}$ is described in the following. The brake pressure is constant $(8 \mathrm{bar})$ and the speed of the motor is varied. The corresponding experimental results are shown in Fig. 9. The red line describes the driving speed, while the blue line represents the torsional vibrations $\Delta \widetilde{\theta}$, where $\Delta \widetilde{\theta}=\Delta \theta-\Delta \theta_{e q}$. The torsional vibration 
of the drive shaft becomes large when creep groan appears. In the accelerating process, the speed is slowly increased from $0.1 \mathrm{rad} / \mathrm{s}$ to $0.8 \mathrm{rad} / \mathrm{s}$, creep groan occurs at a low speed but disappears when the speed is higher than the critical speed $\Omega_{c 12}(0.62 \mathrm{rad} / \mathrm{s}$ with $8 \mathrm{bar})$, which is the boundary point between regions I and II. In the decelerating process, the speed is slowly decreased from $0.8 \mathrm{rad} / \mathrm{s}$ to $0.1 \mathrm{rad} / \mathrm{s}$. Creep groan does not occur at a high speed but appears when the speed is lower than the other critical speed $\Omega_{c 23}(0.42 \mathrm{rad} / \mathrm{s}$ with $8 \mathrm{bar})$, which is the boundary point between regions II and III. It should be noted here that similar boundary points can also be measured by varied pressure with a constant speed (Zhao et al., 2017). For a constant speed with the increasing pressure, the onset of creep groan will occur at a higher brake pressure than the stopping of creep groan will happen when decreasing the brake pressure. This case is considered in Section 5 for the complete real brake.

(a)

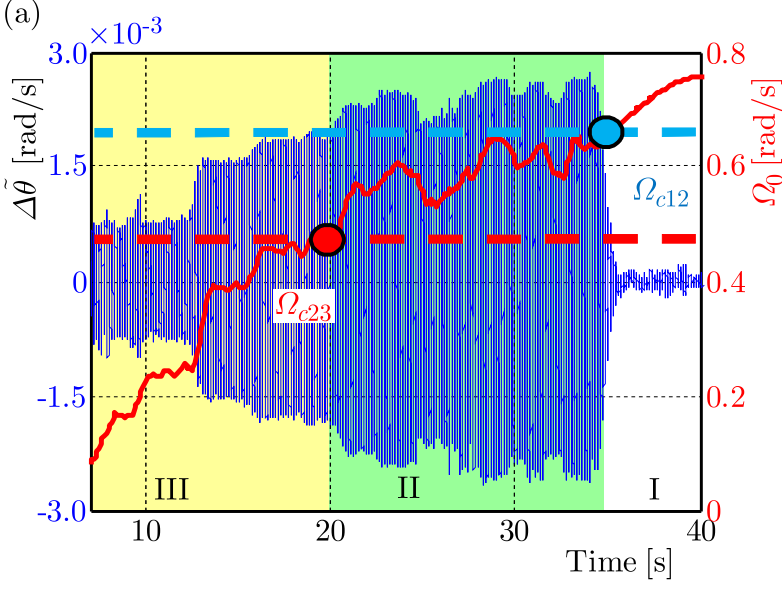

(b)

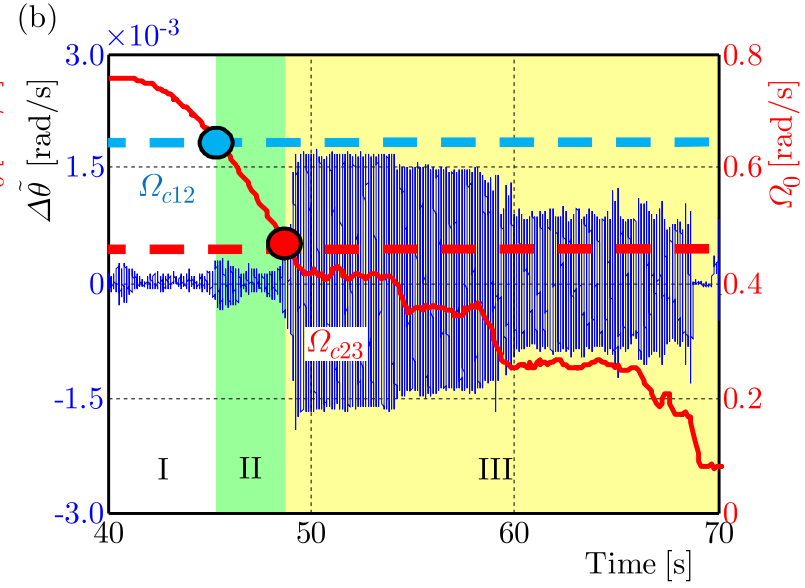

Fig. 9. Experiment: occurrence of creep groan with variation in the speed. Creep groan is always present in region III (yellow) and does not occur in region I (white), while creep groan occurs in region II (green) in the accelerating process (a) but not in the decelerating process (b) cf. Fig. 8

(a)

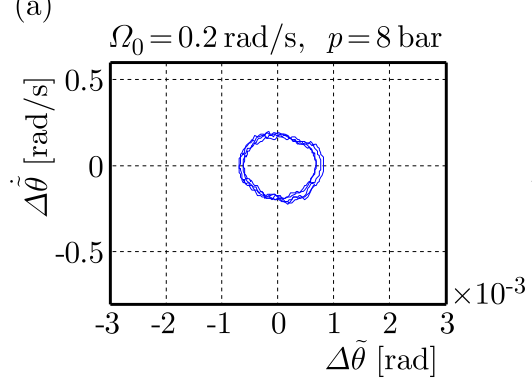

(b)

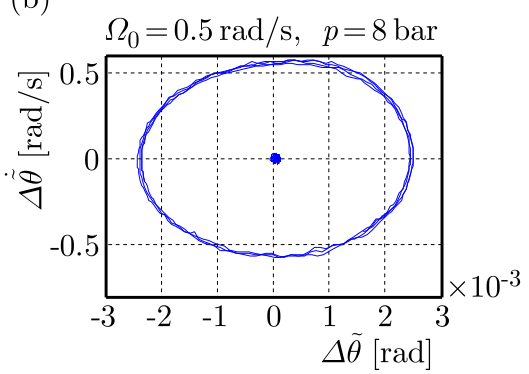

(c)

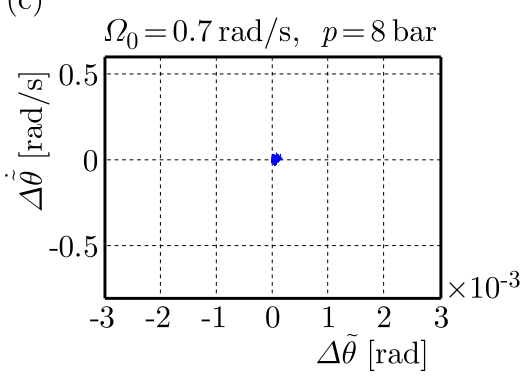

Fig. 10. The equilibrium solution and the stick-slip limit cycle with different driving speeds, (a) in region III with the stick-slip limit cycle, (b) in region II with coexistence of two stable solutions, (c) in region I with just an equilibrium solution

Figure 10 shows the limit cycles and the equilibrium solutions of the system with different speeds in the phase plot. When the speed is higher than $\Omega_{c 12}$ (Fig. 10c), only the equilibrium solution can be measured in both accelerating and decelerating processes. The system under current conditions stays in region I and creep groan cannot occur. When the speed is lower than $\Omega_{c 23}$ (Fig. 10a), only the stick-slip limit cycle can be measured in both processes, meaning that the system is in region III and creep groan always occurs. When the speed is higher than $\Omega_{c 23}$ but lower than $\Omega_{c 12}$ (Fig. 10b), creep groan can be measured in the accelerating process but not in the decelerating process, meaning that the system is in region II with both the stable equilibrium solution and the stable limit cycle, and the occurrence and absence of creep groan depends on its 
initial conditions. The difference between these two limit speed values $\left(\Omega_{c 12}\right.$ and $\left.\Omega_{c 23}\right)$ actually prooves the existence of region II with the coexistence of two stable solutions.

The main result therefore is that a linear stability analysis is not sufficient for the determination of the boundary of creep groan as thereby one can only calculate $\Omega_{c 23}$. E.g., in the acceleration case, which is in fact compared to the deceleration case, the one being more suspicious for creep groan, the boundary $\Omega_{c 12}$ for that undesired vibration phenomenon can only be determined by performing a nonlinear analysis. The same holds in the case of brake pressure variation.

\section{Creep groan bifurcation experimental behavior of a complete real brake}

In this Section, some results on the test rig with a complete real brake will be expressed and compared to the results of the idealized brake. The set-up consists of a real brake system, a suspension system, as well as the same drive system as before, shown in Fig. 11. The similar

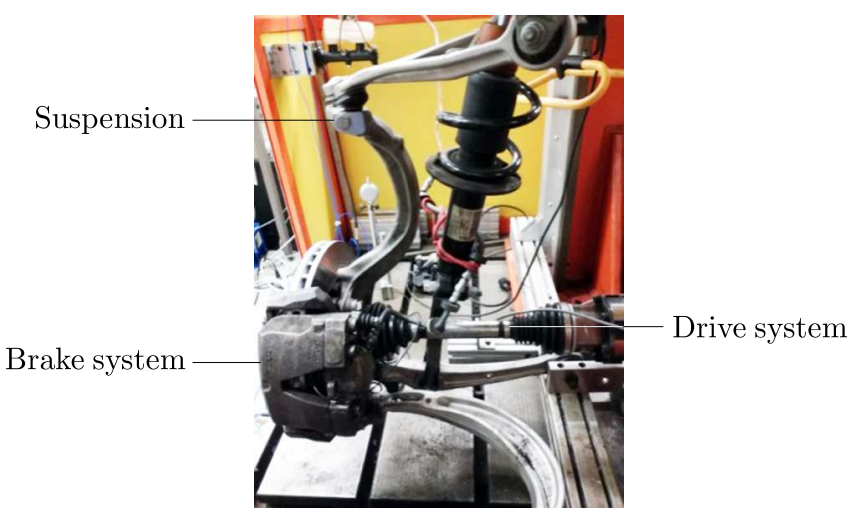

Fig. 11. Test rig with a complete real brake including the suspension
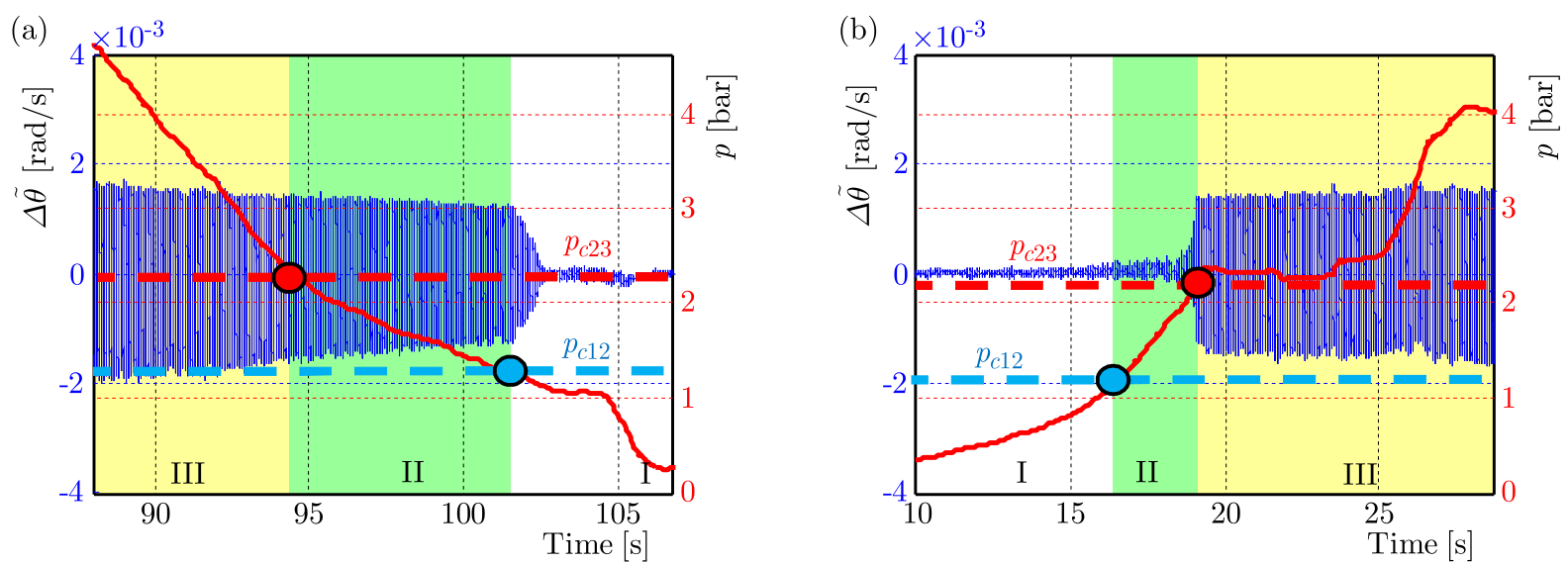

Fig. 12. Occurrence of creep groan with variation of the brake pressure during the pressure decreasing process (a) and pressure increasing process (b). Limits for the stopping and the onset of creep groan ( $p_{c 12}$ and $p_{c 23}$, respectively) are different prooving the existence of region II with two stable solutions, while region III (yellow) and region I (white) contain only one stable solution

experimental method has been used to identify the bifurcation behavior and the corresponding map of creep groan of the complete real brake. According to the above analysis, the boundary between regions I and II is critical for stopping of creep groan in the acceleration process, which is the more suspicious case for creep groan. The boundary between regions II and III is critical 
for the occurrence of creep groan starting from the equilibrium position which is the case while decelerating. We can choose the speed of motor and vary the brake pressure arbitrarily in the test rig and, therefore, the boundaries could be determined. The result for a single test run with decreasing and respectively increasing brake pressure is shown in Fig. 12. Again the boundaries for the stopping and the onset of creep groan are not the same, which prooves the existence of region II with the coexistence of a stable limit cycle and a stable equilibrium solution. The measured boundary points as well as the polynomial regression boundary curves are exhibited in Fig. 13.

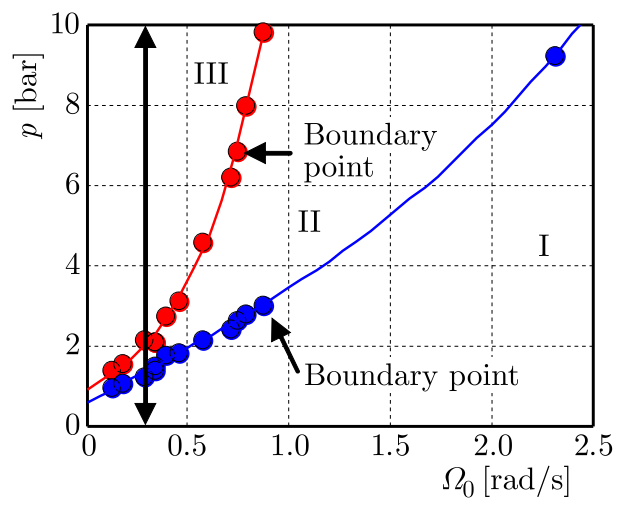

Fig. 13. Map of creep groan identified by experiments, the red points represent the measured boundary points between regions II and III while the blue points represent the measured boundary points between regions I and II, the red line and the blue line are polynomial regression curves of the measured boundary points

Compared to the map measured from the idealized brake, both maps show the same three regions I, II and III, and the boundaries among regions have the same qualitative behavior. It is, therefore, confirmed that the bifurcation behavior observed for the simplified brake can be conveyed in the complete real brake system.

\section{Conclusions}

In this paper, fundamentals of creep groan have been studied theoretically and experimentally. In order to concentrate on the friction contact between the disk and pads, a test rig with an idealized brake has been designed and assembled. Creep groan is measured for this set-up and for the test rig with a complete real brake including suspension.

A nonlinear model with the bristle friction law is proposed to explain the experimental results. Based on the model, creep groan is simulated through a minimal model. Furthermore, the bifurcation behavior and the corresponding map of creep groan are studied. Three parameter regions of the solutions exist in this map at varied brake pressure and driving speed. The parameter regions are denoted as follows: region I with no stick-slip limit cycle but a stable equilibrium solution, region II with both the stable stick-slip limit cycle and the equilibrium solution, and region III with a stable stick-slip limit cycle and an unstable equilibrium solution. The limit cycle can be interpreted as creep groan while the equilibrium solution is the desired vibration free case. In region I, no creep groan can occur, while in region III creep groan is always present. In region II, the occurrence or absence of creep groan depends on the initial conditions. From that, it can be concluded that a linear stability analysis is not sufficient for the determination of the boundary of creep groan. E.g., in the acceleration case, which is in fact compared to the deceleration case, the one being more suspicious for creep groan, the boundary for that undesired vibration phenomenon can only be determined by performing a nonlinear analysis. 
Experimental results confirm the existence of the map with three regions, and the boundaries of the regions can be identified theoretically and experimentally. The simulation results show good agreement with the experimental results. At the end, a similar map is measured in the test rig with a complete real brake, which confirmed that the bifurcation behavior observed for the simplified brake can be conveyed to the complete real brake system.

\section{Acknowledgements}

The first author acknowledges the support by the China Scholarship Council (CSC). The authors thank Dr. Torsten Treyde, ZF TRW, for fruitful discussions and his helpful comments.

\section{References}

1. Abdelhamid M.K., 1995, Creep groan of disc brakes, SAE Technical Paper, 951282, 02-24

2. Abdelhamid M.K., Bray W., 2009, Braking systems creep groan noise: detection and evaluation, SAE Technical Paper, 10-11

3. Antoni J., 2006, The spectral kurtosis: a useful tool for characterising non-stationary signals, Mechanical Systems and Signal Processing, 20, 2, 282-307

4. Antoni J., Randall R., 2006, The spectral kurtosis: application to the vibratory surveillance and diagnostics of rotating machines, Mechanical Systems and Signal Processing, 20, 2, 308-331

5. BRecht J., 2000, Untersuchungen zum Bremsenknarzen-ein Beitrag zur Beschreibung von Schwingungen in Bremssystemen, Shaker, Ph.D. Thesis University Siegen

6. Brecht J., Hoffrichter W., Dohle A., 1997, Mechanisms of brake creep groan, SAE Technical Paper, 106, 2, 3405-3411, DOI: 10.4271/973026

7. Cantoni C., Cesarini R., Mastinu G., Rocca G., Sicigliano R., 2009, Brake comfort - a review, Vehicle System Dynamics, 47, 8, 901-947

8. Crowther A.R., Singh R., 2007, Analytical investigation of stick-slip motions in coupled brakedriveline systems, Nonlinear Dynamics, 50, 3, 463-481

9. Crowther A.R., Singh R., 2008, Identification and quantification of stick-slip induced brake groan events using experimental and analytical investigations, Noise Control Engineering Journal, $\mathbf{5 6}, 4,235-255$

10. De Wit C.C., Olsson H., Astrom K.J., Lischinsky P., 1995, A new model for control of systems with friction, IEEE Transactions on Automatic Control, 40, 3, 419-425

11. Fuadi Z., Adachi K., Ikeda H., Naito H., Kato K., 2009, Effect of contact stiffness on creepgroan occurrence on a simple caliper-slider experimental model, Tribology Letters, 33, 3, 169-178

12. Fuadi Z., Maegawa S., Nakano K., Adachi K., 2010, Map of low-frequency stick-slip of a creep groan, Proceedings of the Institution of Mechanical Engineers. Part J: Journal of Engineering Tribology, 224, 12, 1235-1246

13. Hagedorn P., 1978, Nichtlineare Schwingungen, Akad. Verlag Ges.

14. Hetzler H., Seemann W., Schwarzer D., 2007, Steady-state stability and bifurcations of friction oscillators due to velocity dependent friction characteristics, Proceedings of the Institution of Mechanical Engineers. Part K: Journal of Multi-body Dynamics, 221, 3, 401-412

15. Iвrahim R., 1994a, Friction-induced vibration, chatter, squeal, and chaos. Part I: Mechanics of contact and friction, Applied Mechanics Reviews, 47, 209-226

16. Ibrahim R., 1994b, Friction-induced vibration, chatter, squeal, and chaos. Part II: Dynamics and modeling, Applied Mechanics Reviews, 47, 227-253

17. JANG H., Lee J.S., FASh J.W., 2001, Compositional effects of the brake friction material on creep groan phenomena, Wear, 251, 1, 1477-1483 
18. Johanastrom K., De Wit C.C., 2008, Revisiting the LuGre friction model, IEEE Control Systems, 28, 6, 101-114

19. Muschalle C., 2015, Experimentelle Modalanalyse an einem Versuchsaufbau zur Untersuchung an Kfz-Bremsen, Bachelor thesis, TU Berlin,

20. Neis P.D., Ferreira N.F., Poletto J.C., Matozo L.T., Masotti D., 2016, Quantification of brake creep groan in vehicle tests and its relation with stick-slip obtained in laboratory tests, Journal of Sound and Vibration, 369, 63-76

21. Tustin A., 1947, Effects of backlash and of speed-dependent friction on the stability of closed-cycle control systems, Electrical Engineers - Part IIA: Automatic Regulators and Servo Mechanisms, 94, 1, 143-151

22. VADARI V., JACKSON M., 1999, An experimental investigation of disk brake creep-groan in vehicles and brake dynamometer correlation, SAE Technical Paper, 1999-01-3408

23. von Wagner U., Hochlenert D., Hagedorn P., 2007, Minimal models for disk brake squeal, Journal of Sound and Vibration, 302, 3, 527-539

24. WU G., JIN S., 2014, Combination of test with simulation analysis of brake groan phenomenon, SAE International Journal of Passenger Cars-Mechanical Systems, 7, 1119-1127

25. Zhao X., Gräbner N., von Wagner U., 2016, Experimental and theoretical investigation of creep groan of brakes through minimal models, PAMM, 16, 1, 295-296

26. Zhao X., Gräbner N., von Wagner U., 2017, Creep groan: fundamental experimental and theoretical investigations, Eurobrake, EB2017-FBR-002, Dresden 